\title{
Abdominal Pain and Vomiting in a Patient with Over-anticoagulation -- a Rare Case of Spontaneous Intramural Hematoma of the Jejunum
}

\author{
Dino Kasumović 1 ,*, Matej Nedić ${ }^{1}$, Ana Gudelj-Gračanin ${ }^{1}$, \\ Antonela Šarac ${ }^{1}$, Adrijana Perkunić ${ }^{2}$
}

\author{
${ }^{1}$ Department of Internal Medicine, \\ Clinical Hospital Dubrava, Zagreb, \\ Croatia \\ ${ }^{2}$ Clinical Department of Diagnostic and \\ Interventional Radiology, Clinical \\ Hospital Dubrava, Zagreb, Croatia

\section{*Correspondence \\ dino.kasumovic9@gmail.com, dkasumovic@kbd.hr} \\ (Dino Kasumović)
}

\begin{abstract}
Anticoagulants, including vitamin $\mathrm{K}$ antagonists, are widely used for therapeutic and prophylactic purposes. Bleeding is the most important complication of anticoagulant therapy due to over-anticoagulation. Over-anticoagulation may present in unusual ways, such as spontaneous intramural hematoma of the small bowel. The classical clinical picture consists of abdominal pain, small bowel obstruction (that can present as vomiting) and hemorrhagic symptoms that can be related to the bowel or other parts of the body. Radiological examinations are essential for the diagnosis. Ultrasound can be helpful in the diagnostic process, but computed tomography is the procedure of choice. Conservative treatment is usually successful, including procedures that stop the over-anticoagulant consequences. A surgical approach is reserved for complications such as necrosis or perforation of the bowel. The diagnosis is definitively confirmed by the spontaneous resolution of the pathological findings on a follow-up computed tomography scan. We present a case of a 72-year-old woman who was diagnosed with this rare condition affecting the jejunum in the emergency department in our hospital.
\end{abstract}

\section{Keywords}

Anticoagulants, Hematoma, Jejunum, Abdominal pain, Computed tomography

\section{Introduction}

Gastrointestinal bleeding is the most common adverse event associated with the use of oral anticoagulants such as warfarin [1]. One rare presentation of this condition is spontaneous intramural gastrointestinal tract hematoma. The small bowel is affected in up to $85 \%$ of cases, with the jejunum being the most affected region; in post-traumatic cases, the duodenum is most commonly affected [2]. Although this presentation is very rare, the number of reported cases has increased recently. One reason is the availability of current radiologic diagnostic methods, primarily computed tomography (CT); another is the growing number of patients on anticoagulant therapy [3]. The most commonly reported symptoms are abdominal pain, nausea and vomiting (due to high intestinal obstruction) [2]. We present a case of a woman presenting with these symptoms in our emergency department, who was later diagnosed with intramural jejunal hematoma.

\section{Case report}

A 72-year-old Caucasian woman presented to the emergency department with occasional vomiting that lasted for 2 days. On the day she sought help, she started experiencing abdominal pain throughout the abdomen. The abdomen was distended, soft and diffusely painful on palpation, and bowel sounds were heard. Further examination revealed sinus tachycardia, while she was normotensive and afebrile. She had a medical history of arterial hypertension and paroxysmal atrial fibrillation for which she had been taking warfarin for 53 months. Laboratory tests revealed mild normocytic anemia (hemoglobin $112 \mathrm{~g} / \mathrm{L}$ [ref. $119-157 \mathrm{~g} / \mathrm{L}$ ]), acute kidney injury (urea $24 \mathrm{mmol} / \mathrm{L}$ [ref. $2.8-8.3 \mathrm{mmol} / \mathrm{L}$ ], and creatinine $241 \mu \mathrm{mol} / \mathrm{L}$ [ref. 49 - $90 \mu \mathrm{mol} / \mathrm{L}])$. The urine test showed microhematuria $(>$ 100 erythrocytes per high power field [ref. 0 - 2] in urine sediment). The most striking finding was an excessively prolonged prothrombin time (international normalized ratio $>$ 6.0 [ref. 2 - 3]) due to over-anticoagulation with warfarin. Plain abdominal X-ray showed no pathological signs. Since the abdomen was distended and she had a history of vomiting, a nasogastric tube was applied to inspect the gastric material, and it showed miserere. This indicated further investigations; CT showed a moderate amount of free liquid with a dense content intraperitoneally and a high-density liquid in the pelvis, which was probably hemorrhagic. As the patient reported no trauma, further findings (Fig. 1) indicated spontaneous intramural hematoma of the jejunum with additional hematoma in the retroperitoneum (Fig. 2). The patient was examined by a surgeon, but no criteria for urgent operation were found. She initially received $1500 \mathrm{ml}$ saline solution, $20 \mathrm{mg}$ parenteral 


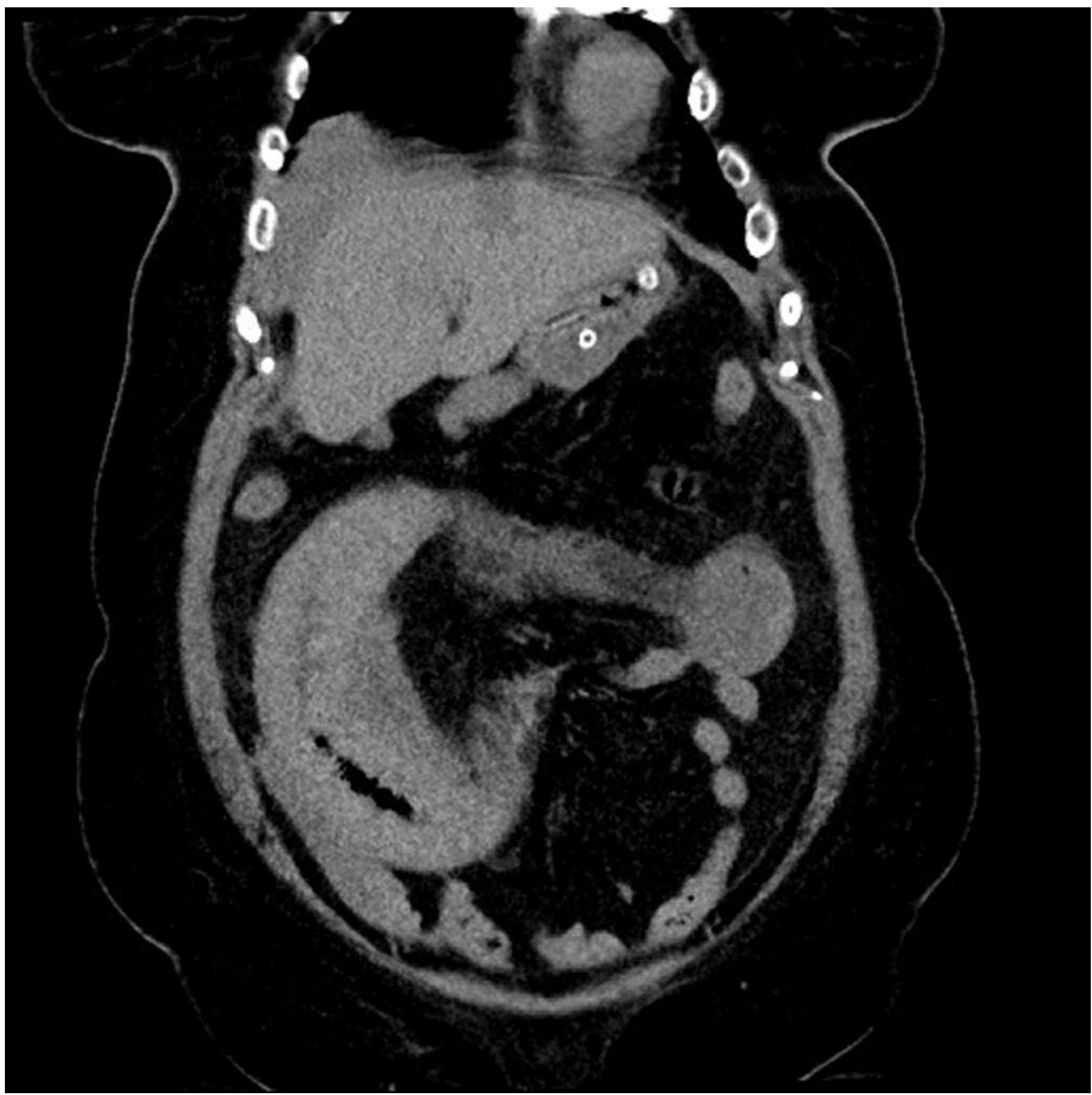

F I G U R E 1. A circumferentially thickened (up to $1.5 \mathrm{~cm}$ ) hyperdense wall of an extended proximal section of the jejunum (arrow), which appeared up to $4 \mathrm{~cm}$ in diameter, with accentuated mesentery and blurring of the surrounding fatty tissue.

vitamin $\mathrm{K}$ and 2 fresh frozen plasma transfusions while warfarin was withheld. She was admitted to the intensive care unit. After being continuously hemodynamically stable, she was soon transferred to a nonintensive ward. A second CT was performed one week after the first CT, showing that all hemorrhagic content was disappearing. She was discharged after 10 days of hospitalization in good condition with complete recovery of renal function. After 3.5 months, a follow-up CT was performed, showing total regression of all hematomas. She is waiting for a cardiologist appointment regarding her anticoagulant therapy.

\section{Discussion}

Although over-anticoagulation is the most common cause of spontaneous intramural hematoma of the jejunum, other conditions, such as hemophilia or cirrhosis of the liver, can also cause this complication [4]. Sorbello et al. reviewed articles with a total of 57 cases. The incidence was higher in males $(60 \%)$ than in females, and the average age at diagnosis was 57.6 years. The average time from symptom onset until medical attention was 2.5 days. The most frequent clinical presentation was abdominal pain ( $97.5 \%$ of cases), with nausea present in half and vomiting $40 \%$ of cases. Gastrointestinal hemorrhage as a result of ruptured hematoma can also occur, 


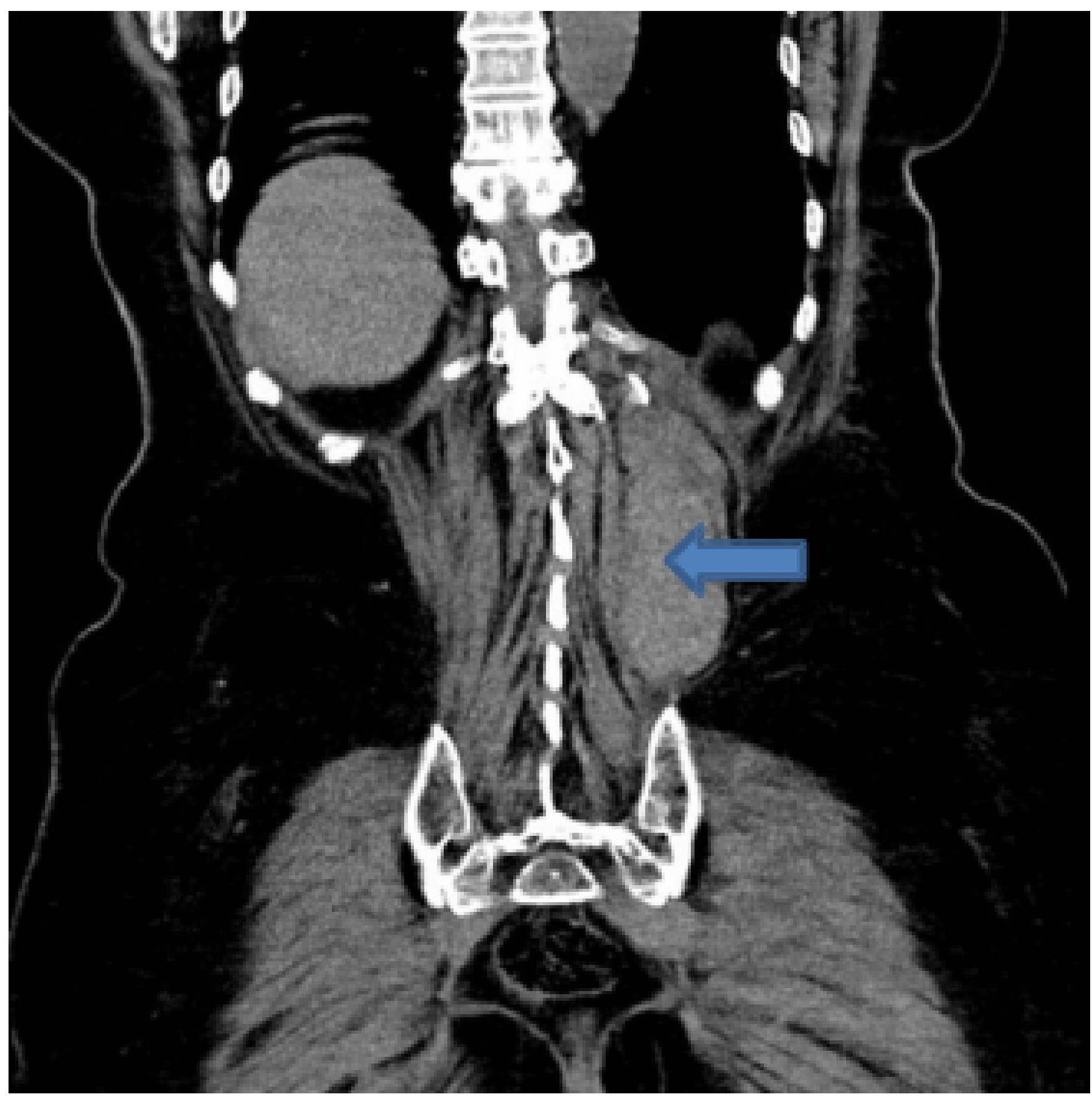

F I G U R E 2. Hematoma of the paravertebral muscles (arrow) on the left side (length $20 \mathrm{~cm}$, axial width $6 \mathrm{~cm}$ ).

but obvious bleeding was present in less than half of the cases. Some cases were related to additional extraintestinal bleeding, such as epistaxis and hematuria [3]. Our patient had concomitant intraperitoneal, pelvic and retroperitoneal hemorrhage (paravertebral muscles) and microhematuria. Signs of peritoneal irritation may be present and usually indicate the development of complications, such as necrosis or perforation of the bowel [5]. Although the presence of anemia at the time of admission is an important finding, it should not be considered defining. However, a sudden decrease in hemoglobin levels may point to serious bleeding [6]. CT is the diagnostic exam of choice, and has been shown to be extremely sensitive [3]. Characteristic findings on CT include circumferential wall thickening with intramural hyperdensity and luminal narrow- ing with intestinal obstruction [7]. The diagnosis is definitively confirmed by the spontaneous resolution of the pathological findings on follow-up CT [4]. Although less specific, ultrasound can also be helpful; Polat et al. demonstrated that a combination of ultrasound and CT showed the exact pathology in all covered cases [8]. Plain abdominal X-ray may not show evidence of intestinal obstruction, which can be misleading in the diagnostic process [9]. Due to the rarity of intramural bowel hematoma, there are no studies with sufficient evidence to standardize treatment. These following steps should be undertaken when the diagnosis is caused by warfarin overanticoagulation: termination of the drug and oral food intake, fresh frozen plasma transfusion and vitamin $\mathrm{K}$ administration, erythrocyte transfusion in patients with anemia, nasogastric 
decompression in patients with vomiting, and daily followup of blood count, clotting parameters and nutrition needs $[6,9]$. Surgery is required only for uncertain diagnoses or complications [9]. Reinitiation of anticoagulation in patients who recently had significant bleeding events is often clinically justified [10]. In uncomplicated cases such as ours, regression of the hematomas tends to occur within a week [5], and complete resolution usually occurs within two months after onset [9]. Small bowel lesions persisting for more than 2 months should raise the suspicion for other underlying conditions, such as neoplasms or inflammatory bowel disease [4].

\section{Conclusion}

Patients complaining of abdominal pain and/or vomiting are frequently seen in emergency departments. If the patient is taking a vitamin $\mathrm{K}$ antagonist, the prothrombin time should always be assessed, even when no visible signs of bleeding are present. This sign can be hidden, as in cases of spontaneous intramural hematoma of the jejunum, which can usually be managed conservatively.

\section{ACKNOWLEDGMENTS}

I would like to express my gratitude to all those who helped me during the writing of this manuscript.

\section{CONFLICT OF INTEREST}

We declare that we do not have any commercial or associative interest that represents a conflict of interest in connection with the work submitted.

\section{REFERENCES}

[1] Lanas-Gimeno A, Lanas A. Risk of gastrointestinal bleeding during anticoagulant treatment. Expert Opin Drug Saf. 2017;16:673-685.

[2] Abdel Samie A, Theilmann L. Detection and management of spontaneous intramural small bowel hematoma secondary to anticoagulant therapy. Expert Rev Gastroenterol Hepatol. 2012;6:553-559.

[3] Sorbello MP, Utiyama EM, Parreira JG, et al. Spontaneous intramural small-bowel hematoma induced by anticoagulant therapy: review and case report. Sao Paulo. 2007;62:785-790.

[4] Abbas MA, Collins JM, Olden KW. Spontaneous intramural small-bowel hematoma: imaging findings and outcome. AJR Am J Roentgenol. 2002;179:1389-1394.

[5] Abdel Samie A, Sun R, Huber A, et al. Spontaneous intramural smallbowel hematoma secondary to anticoagulant therapy: a case series. Med Klin Intensivmed Notfmed. 2013;108:144-148.

[6] Altintoprak F, Dikıcıer E, Akyüz M, et al. A retrospective review of patients with non-traumatic spontaneous intramural hematoma. Turk J Gastroenterol. 2013;24:392-399.

[7] Cheng J, Vemula N, Gendler S. Small bowel obstruction caused by intramural hemorrhage secondary to anticoagulant therapy. Acta Gastroenterol Belg. 2008;71:342-344.

[8] Polat C, Dervisoglu A, Guven H, et al. Anticoagulant-induced intramural intestinal hematoma. Am J Emerg Med. 2003;21:208-211.

[9] Carkman S, Ozben V, Saribeyoğlu K, et al. Spontaneous intramural hematoma of the small intestine. Ulus Travma Acil Cerrahi Derg. 2010;16:165-169.

[10] Staerk L, Lip GY, Olesen JB, et al. Stroke and recurrent haemorrhage associated with antithrombotic treatment after gastrointestinal bleeding in patients with atrial fibrillation: nationwide cohort study. BMJ. 2015;351:5876.

How to cite this article: Dino Kasumović, Matej Nedić, Ana GudeljGračanin, Antonela Šarac, Adrijana Perkunić. Abdominal Pain and Vomiting in a Patient with Over-anticoagulation - a Rare Case of Spontaneous Intramural Hematoma of the Jejunum. Signa Vitae. 2020;16(2):203-206. doi:10.22514/sv.2020.16.0035. 